\title{
Wheeled Mobile Robot RBFNN Dynamic Surface Control Based on Disturbance Observer
}

\author{
Shaohua Luo, ${ }^{1,2}$ Songli Wu, ${ }^{2}$ Zhaoqin Liu, ${ }^{2}$ and Hao Guan' \\ ${ }^{1}$ State Key Laboratory of Mechanical Transmission, Chongqing University, Chongqing 400044, China \\ ${ }^{2}$ Department of Mechanical Engineering, Chongqing Aerospace Polytechnic College, Chongqing 400021, China
}

Correspondence should be addressed to Shaohua Luo; hua66com@163.com

Received 13 November 2013; Accepted 9 December 2013; Published 11 February 2014

Academic Editors: X.-G. Yan and X.-S. Yang

Copyright (C) 2014 Shaohua Luo et al. This is an open access article distributed under the Creative Commons Attribution License, which permits unrestricted use, distribution, and reproduction in any medium, provided the original work is properly cited.

This paper focuses on the problem of an adaptive neural network dynamic surface control (DSC) based on disturbance observer for the wheeled mobile robot with uncertain parameters and unknown disturbances. The nonlinear observer is used to compensate for the external disturbance, and the neural network is employed to approximate the uncertain and nonlinear items of system. Then, the Lyapunov theory is introduced to demonstrate the stabilization of the proposed control algorithm. Finally, the simulation results illustrate that the proposed algorithm not only is superior to conventional DSC in trajectory tracking and external friction disturbance compensation but also has better response, adaptive ability, and robustness.

\section{Introduction}

Wheeled mobile robot is an intelligent object. It can collect the surrounding environmental information from the constant feedback of sensors and allodial makes decisions. Then, it outputs motion instructions and guides itself to move to the destination quickly with high precision of trajectory tracking $[1,2]$. However, there still exists a challenging issue to control robot to obtain perfect dynamic performance because its mathematical model is usually multivariable, coupled, and nonlinear. Dong and Kuhnert [3] presented a tracking control approach for mobile robots with both parameter and nonparameter uncertainties. In [4], an wavelet-network-based controller is developed for mobile robots with unstructured dynamics and disturbances. Chwa [5] designed a position and heading direction controller using the SMC method for nonholonomic mobile robots. $\mathrm{Gu}$ and $\mathrm{Hu}$ [6] studied the receding horizon tracking control on the wheeled mobile robots, which used the optimized method to accelerate the convergence speed of errors.

Nowadays various intelligence control algorithms for mobile robot have been represented in the literature, such as genetic algorithm [7], iterative learning control [8], neural networks [9], fuzzy logic [10], and backstepping. In the above-mentioned methods, the backstepping method is preferred. In [11], an adaptive tracking controller using a backstepping method is presented for the dynamic model of mobile robots with unknown parameters. Unfortunately, the backstepping suffers from the "explosion of complexity" caused by the repeated differentiation of virtual control functions [12]. Dynamic surface control (DSC) $[13,14]$ is a new control technique by introducing a first-order filter at each recursive step of the backstepping design procedure, such that the differentiation item on the virtual function can be avoided. Zhang and Ge further studied the control design for some special nonlinear systems with time delay or dead zone using the DSC technique [15]. However, the adaptive parameters involved in the aforementioned DSC still impose that a large number of parameters need to be tuned online in the NN approximation. In addition, a robust adaptive DSC for a class of uncertain perturbed strict-feedback nonlinear systems preceded by unknown backlash-like hysteresis is proposed [16].

On the other hand, the output of the disturbance observer can be extensively used in feed-forward compensation of external disturbances. The disturbance observers can give fast, excellent tracking performance and smooth control actions without the use of large feedback gains [17]. Zhongyi 
et al. [18] presented a disturbance observer-based control scheme for free-floating space manipulator with nonlinear dynamics derived using the virtual manipulator approach. Yan presented a nonlinear dynamic output feedback decentralized controller and further studied state and parameter estimation for nonlinear delay systems using sliding mode observer $[19,20]$. The friction compensation schemes based on disturbance observer is in that they are not based on any particular friction models [21].

To the best of our knowledge, the DSC method and disturbance observer have been seldom used in the control of wheeled mobile robot so far. The first main contribution of this paper is the compensation of external friction by using the nonlinear disturbance observer. The second contribution is the design of the dynamic surface control method combined with neural network. During the design process, neural networks are employed to approximate the nonlinearities, and adaptive method and DSC are used to construct neural network controller. It means that uncertain parameters are taken into account and explosion of complexity is solved. Finally, to testify to the superiority of the proposed control algorithm, a comparison between DSC, neural network dynamic surface controller (NNDSC) and NNDSC with nonlinear observer is studied. The simulation results are provided to demonstrate the effectiveness and robustness objectively against the parameter uncertainties and external disturbances.

\section{Kinetic Models}

2.1. Mathematical Model of Wheeled Mobile Robot. The schematic diagram of wheeled mobile robot is shown in Figure 1. It has two independent actuated rear-wheels with servo motor driven. To change the relative input voltage to realize speed difference of two rear-wheels, it is achieved to adjust the position of both car body and tracking trajectory. Front wheels only support the car body as supporting roller.

The dynamics of wheeled mobile robot with uncertain parameters and nonlinearity in Figure 1 is generally described by [22]:

$$
\begin{gathered}
\dot{v}=-\frac{2 c}{M r^{2}+2 I_{w}} v+\frac{k r}{M r^{2}+2 I_{w}}\left(u_{r}+u_{l}\right), \\
\ddot{\phi}=-\frac{2 c L^{2}}{I_{v} r^{2}+2 I_{w} L^{2}} \dot{\phi}+\frac{k r L}{I_{v} r^{2}+2 I_{w} L^{2}}\left(u_{r}-u_{l}\right), \\
k=\frac{i k_{m}}{R_{a}},
\end{gathered}
$$

where $I_{v}$ stands for the moment of inertia around the center of gravity of robot, $I_{w}$ denotes the moment of inertia of wheel, $M$ represents the mass of robot, $k$ stands for the driving gain factor, $r$ denotes the radius of wheel, $c$ represents the viscous friction factor of both wheel and ground, $u_{r}$ and $u_{l}$ stand for the right and left driving input of rear axle, respectively, $R_{a}$ denotes the armature resistance of motor, $k_{m}$ represents the electromagnetism torque constant of motor, $i$ stands for the transmission ratio of reducer, $v$ denotes the velocity of robot, and $\varphi$ represents the azimuth of robot.

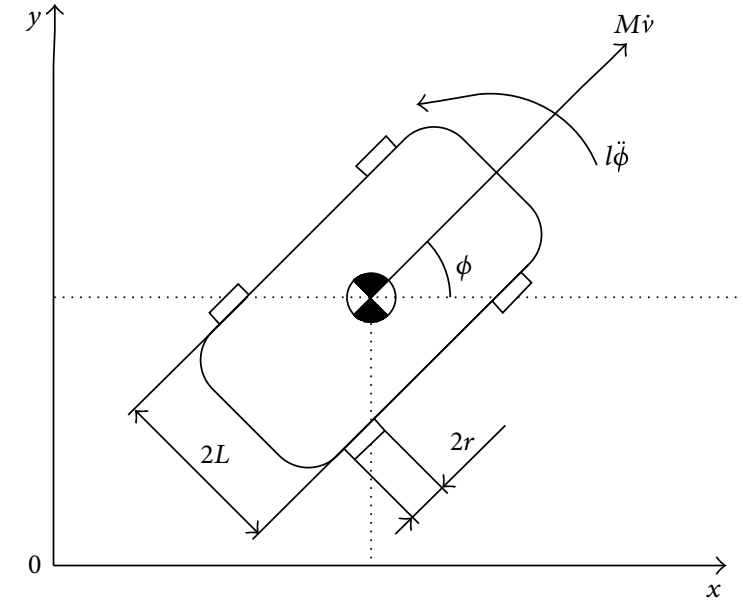

Figure 1: The wheeled mobile robot.

For simplicity, the following notations are introduced:

$$
\begin{gathered}
x_{1}=v, \quad x_{2}=\phi, \quad x_{3}=\dot{x}_{2}, \\
a_{1}=-\frac{2}{M r^{2}+2 I_{w}}, \quad b_{1}=\frac{r}{M r^{2}+2 I_{w}}, \\
a_{2}=-\frac{2 L^{2}}{I_{v} r^{2}+2 I_{w} L^{2}}, \quad b_{2}=\frac{r L}{I_{v} r^{2}+2 I_{w} L^{2}} .
\end{gathered}
$$

Outdoor wheeled mobile robot suffers more unknown and uncertain disturbance than indoor one. In the real control system it is unavoidable for some implicit, prior unknown modeling and external disturbance to exist. Suppose the sum of all external uncertainty items to describe with function $d$. Uncertainties consisted of gear clearance existence of reducer in the transmission system, friction coefficient variation of roadbed and motor parameter change because of ambient temperature surrounded, material wear, and so on, influence static, and dynamic performances of robot to some extent. Therefore, the drive gain $k$ and friction coefficient $c$ are uncertain.

By using these notations, the dynamic model of wheeled mobile robot can be described by the following differential equations:

$$
\begin{gathered}
\dot{x}_{1}=a_{1} c x_{1}+b_{1} k\left(u_{r}+u_{l}\right), \\
\dot{x}_{2}=x_{3}, \\
\dot{x}_{3}=a_{2} c x_{3}+b_{2} k\left(u_{r}-u_{l}\right)+d .
\end{gathered}
$$

Remark 1. $\quad d$ is unknown, but its upper bound is $|d(\dot{\phi}, \phi, t)| \leq$ $\delta$ and $\delta \geq 0$.

2.2. System Decoupling. System dynamic equations of wheeled mobile robot are a coupled system. Firstly it is to decouple the system mentioned and then to convert to parametric strict-feedback forms. It is defined as follows:

$$
\left[\begin{array}{l}
u_{r} \\
u_{l}
\end{array}\right]=\left[\begin{array}{cc}
1 & -1 \\
0 & 1
\end{array}\right]\left[\begin{array}{l}
u_{1} \\
u_{2}
\end{array}\right]
$$


Then $u_{r}=u_{1}-u_{2}$; (3) can successfully decouple two independent subsystems below:

$$
\begin{gathered}
\dot{x}_{1}=a_{1} c x_{1}+b_{1} k u_{1}, \\
\dot{x}_{2}=x_{3}, \\
\dot{x}_{3}=a_{2} c x_{3}+b_{2} k u_{1}-2 b_{2} k u_{2}+d .
\end{gathered}
$$

Assumption 2. The reference signals $y_{r}$ and $y_{d}$ are continuous about $t$ and satisfy $\left(y_{i}, \dot{y}_{i}, \ddot{y}_{i}\right) \in \Xi_{i}, i=r, d$, where $\Xi_{i}=$ $\left\{\left(y_{i}, \dot{y}_{i}, \ddot{y}_{i}\right): y_{i}^{2}+\dot{y}_{i}^{2}+\ddot{y}_{i}^{2} \leq B_{i}\right\}$ and $B_{i}$ is the known positive constant.

\section{RBFNN Dynamic Surface Controller Based on Nonlinear Disturbance Observer}

3.1. RBF Neural Network. Figure 2 shows the RBF neural network expression which is given as follows:

$$
f_{m}(\theta, z)=\theta^{T} \xi(z),
$$

where $z \in \Omega \subset R^{n}$ is the neural network input vector, $\Omega$ denotes some compact set in $R^{n}, \theta \in R^{l}$ is the neural network weight vector, $l$ is the node number of neuron, and $\xi(z)=$ $\left[\xi_{1}(z), \xi_{2}(z), \ldots, \xi_{n}(z)\right]^{T} \in R^{T}$, where $\xi_{i}(z)$ is basic function. Then Gaussian function can be formulated as

$$
\begin{aligned}
\xi_{i}(z) & =\exp \left[-\frac{\left|z-\mu_{i}\right|^{2}}{\sigma_{i}^{2}}\right] \\
& =\exp \left[-\frac{\left(z-\mu_{i}\right)^{T}\left(z-\mu_{i}\right)}{\sigma_{i}^{2}}\right], \quad i=1,2, \ldots, l,
\end{aligned}
$$

where $\mu_{i}=\left[\mu_{i 1}, \mu_{i 2}, \ldots, \mu_{i n}\right]^{T}$ is the center of basic function $\xi_{i}(z)$ and $\sigma_{i}$ is the width of $\xi_{i}(z)$.

Remark 3. For the Gaussian neural network, $\Omega_{z} \subset R^{n}$, and $f(\cdot)$ stands for the real value continuous function in the field of $\Omega_{z}$. Therefore, for any given $\varepsilon>0$, there always exist positive integer $l$ and constant vector $\theta$ such that

$$
\max _{z \in \Omega_{z}}\left|f(z)-f_{m}(\theta, z)\right|<\varepsilon .
$$

3.2. Nonlinear Disturbance Observer. Define state variable $X=\left[x_{2}, x_{3}\right]^{T}$. Using (6), it is constructed as

$$
\dot{X}=f(X)+B(X) u_{2}+C(X) d,
$$

where $f=\left[\begin{array}{c}x_{3} \\ a_{2} c x_{3}+b_{2} k u_{1}\end{array}\right], B=\left[\begin{array}{c}0 \\ -2 b_{2} k\end{array}\right]$, and $C=\left[\begin{array}{l}0 \\ 1\end{array}\right]$.

By equation transformation, (10) can be rewritten as

$$
C d=\dot{X}-f-B u_{2} .
$$

On the basis of the difference between real output and evaluated output of system, the evaluator can be adjusted to design disturbance observer.

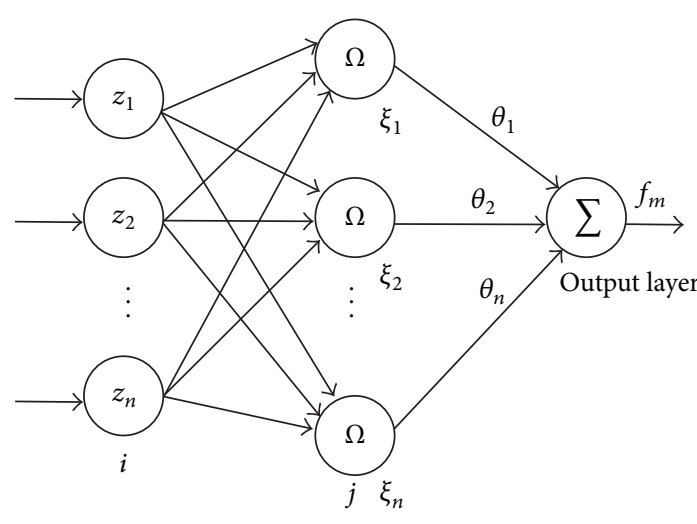

Input layer

Hidden layer

FIGURE 2: A general architecture of RBF neural network.

Suppose that nonlinear disturbance observer owns the following form:

$$
\dot{\grave{d}}=L\left(\dot{X}-f-B u_{2}-C \widehat{d}\right),
$$

where $\widehat{d}$ is estimated value of $d$.

Define the auxiliary variable:

$$
Z=\widehat{d}-u_{2} .
$$

The gain $L$ of nonlinear disturbance observer can be described by the following differential equation:

$$
L=\frac{\partial u_{2}(X)}{\partial X} .
$$

Differentiating $Z$ gives

$$
\begin{aligned}
\dot{Z} & =\dot{\bar{d}}-\dot{u}_{2}=L\left(-f-B u_{2}-C \widehat{d}\right) \\
& =L\left(-f-B u_{2}-C\left(Z+u_{2}\right)\right) .
\end{aligned}
$$

Define the error as $e_{d}=d-\widehat{d}$, and the time derivative of $e_{d}$ is computed by

$$
\dot{e}_{d}+L C e_{d}=-\dot{\bar{d}}+L C(d-\widehat{d}) .
$$

Substituting (12) into (16) gives

$$
\dot{e}_{d}+L C e_{d}=-L\left(\dot{X}-f-B u_{2}-C d\right)=0 .
$$

Introduce gain constant vector $L=\left[c_{1}, c_{2}\right]$; then

$$
u_{2}=c_{1} x_{2}+c_{2} x_{3} \text {, }
$$

At this present stage, nonlinear disturbance observer is designed as

$$
\begin{gathered}
\hat{d}=Z+c_{1} x_{2}+c_{2} x_{3} \\
\dot{Z}=-c_{1} x_{3}+c_{2}\left[-a_{2} c x_{3}-b_{2} k u_{1}+2 b_{2} k u_{2}\right. \\
\left.-Z-c_{1} x_{2}-c_{2} x_{3}\right] .
\end{gathered}
$$


3.3. Neural Network Dynamic Surface Controller with Disturbance Compensation. By obtaining the output of neural network dynamic surface controller, the disturbance of wheeled mobile robot existing is compensated well. The control law is designed as

$$
u_{2}=u_{\mathrm{ND}}-u_{D}
$$

where $u_{\mathrm{ND}}$ is output of neural network dynamic surface controller and $u_{D}$ is output of nonlinear disturbance observer.

Furthermore, (10) yields

$$
\dot{x}_{3}=a_{2} c x_{3}+b_{2} k u_{1}-2 b_{2} k\left(u_{\mathrm{ND}}-u_{D}\right)+d .
$$
that

Define $u_{D}=-1 / 2 b_{2} k \cdot \hat{d}$, and then substitute it into (21)

$$
\dot{x}_{3}=a_{2} c x_{3}+b_{2} k u_{1}-2 b_{2} k u_{\mathrm{ND}}+\tilde{d} .
$$

To aim at the wheeled mobile robot represented in (5) and (22), the designing steps of neural network dynamic surface controller are described as follows.

Step 1. For the reference signal $y_{d}$, define the dynamic surface as $S_{1}=x_{1}-y_{d}$. Obviously, the time derivative of $S_{1}$ is given by

$$
\dot{S}_{1}=a_{1} c x_{1}+b_{1} k u_{1}-\dot{y}_{d}=f_{1}+b_{1} k u_{1},
$$

where $f_{1}\left(S_{1}\right)=a_{1} c x_{1}-\dot{y}_{d}$. Notice that $f_{1}$ contains the derivative of $y_{d}$ and unknown friction coefficient $c$. This will make the classical dynamic surface control become very complex and troublesome. Therefore, we will employ the neural network to approximate the function $f_{1}$. According to the description above, there exists a neural network system such that

$$
f_{1}\left(S_{1}\right)=\theta_{1}^{T} \xi_{1}\left(S_{1}\right)+\delta_{1}\left(S_{1}\right),
$$

where $\delta_{1}$ is the approximation error and satisfies $\left|\delta_{1}\left(S_{1}\right)\right|<\varepsilon_{1}$.

According to (23) and (24), the corresponding control law is chosen as follows:

$$
u_{1}=\frac{\widehat{k}}{\left(\hat{k}^{2}+\varepsilon\right) b_{1}}\left(-\widehat{\theta}_{1}^{T} \xi_{1}-k_{1} S_{1}\right),
$$

where $\widehat{k}$ and $\widehat{\theta}_{1}$ are the evaluated value of $k$ and $\theta_{1}$ at the time of $t$, respectively, and $k_{1}>0$ is a design constant.

The adaptive laws are chosen as

$$
\begin{aligned}
& \dot{\vec{\theta}}_{1}=\eta_{1}\left(\xi_{1} S_{1}-m_{1} \widehat{\theta}_{1}\right), \\
& \dot{\widehat{k}}=\eta_{3}\left(S_{1} u_{1}-m_{3} \widehat{k}\right),
\end{aligned}
$$

where $\eta_{1}, \eta_{3}, m_{1}$, and $m_{3}$ are the positive design parameters.

Step 2. For the reference signal $y_{r}$, define the dynamic surface as $S_{2}=x_{2}-y_{r}$. Obviously, the time derivative of $S_{2}$ is given by

$$
\dot{S}_{2}=x_{3}-\dot{y}_{r}
$$

Construct the virtual control law $\alpha$ as

$$
\alpha=-k_{2} S_{2}+\dot{y}_{r}
$$

with $k_{2}>0$ being a design parameter.

According to the known $\alpha, \alpha_{f}$ is calculated by the firstorder filter:

$$
\tau \dot{\alpha}_{f}+\alpha_{f}=\alpha, \quad \alpha_{f}(0)=\alpha(0),
$$

where $\tau$ is a positive filter time constant.

Step 3. Define the dynamic surface as $S_{3}=x_{3}-\alpha_{f}$; the time derivative of $S_{3}$ is given by

$$
\begin{aligned}
\dot{S}_{3} & =a_{2} c x_{3}+b_{2} k u_{1}-2 b_{2} k u_{\mathrm{ND}}+\tilde{d}-\dot{\alpha}_{f} \\
& =f_{2}+b_{2} k u_{1}-2 b_{2} k u_{\mathrm{ND}}-\dot{\alpha}_{f},
\end{aligned}
$$

where $f_{2}\left(S_{3}\right)=a_{2} c x_{3}+\tilde{d}$. Notice that $f_{2}$ contains unknown $\widetilde{d}$ and uncertainty friction coefficient $c$. To make full use of universal approximation theorem of neural network, for any given $\varepsilon_{2}>0$, there is a neural network $\widehat{\theta}_{2}^{T} \xi_{2}\left(S_{3}\right)$ such that

$$
f_{2}\left(S_{3}\right)=\theta_{2}^{T} \xi_{2}\left(S_{3}\right)+\delta_{2}\left(S_{3}\right)
$$

where $\delta_{2}$ is the approximation error and satisfies $\left|\delta_{2}\right| \leq \varepsilon_{2}$.

Combining (30) and (31), design control law as

$$
u_{\mathrm{ND}}=\frac{\widehat{k}}{\left(\hat{k}^{2}+\varepsilon\right) 2 b_{2}}\left(\widehat{\theta}_{2}^{T} \xi_{2}+k_{3} S_{3}+b_{2} \widehat{k} u_{1}-\dot{\alpha}_{f}\right) \text {, }
$$

where $\widehat{\theta}_{2}$ is the estimation of $\theta_{2}$ at the time of $t$ and $k_{3}>0$ is a design constant.

The adaptive laws are chosen as

$$
\begin{aligned}
& \dot{\vec{\theta}}_{2}=\eta_{2}\left(\xi_{2} S_{3}-m_{2} \widehat{\theta}_{2}\right), \\
& \dot{\widehat{k}}=\eta_{3}\left(S_{1} u_{1}-m_{3} \widehat{k}\right) .
\end{aligned}
$$

From the analysis mentioned above, the control schematic of wheeled mobile robot neural network dynamic surface control based on nonlinear disturbance observer is shown clearly in Figure 3.

3.4. A Comparison with Traditional Dynamic Surface Control. To verify the proposed controller, we make a comparison between neural network dynamic surface control and traditional dynamic surface control.

Step 1. From (23), the control law is chosen as

$$
u_{1}=\frac{1}{b_{1} k}\left(-k_{1} S_{1}-a_{1} c x_{1}+\dot{y}_{d}\right) .
$$

Step 2. According to the definition, the virtual control function is equal to (28). 


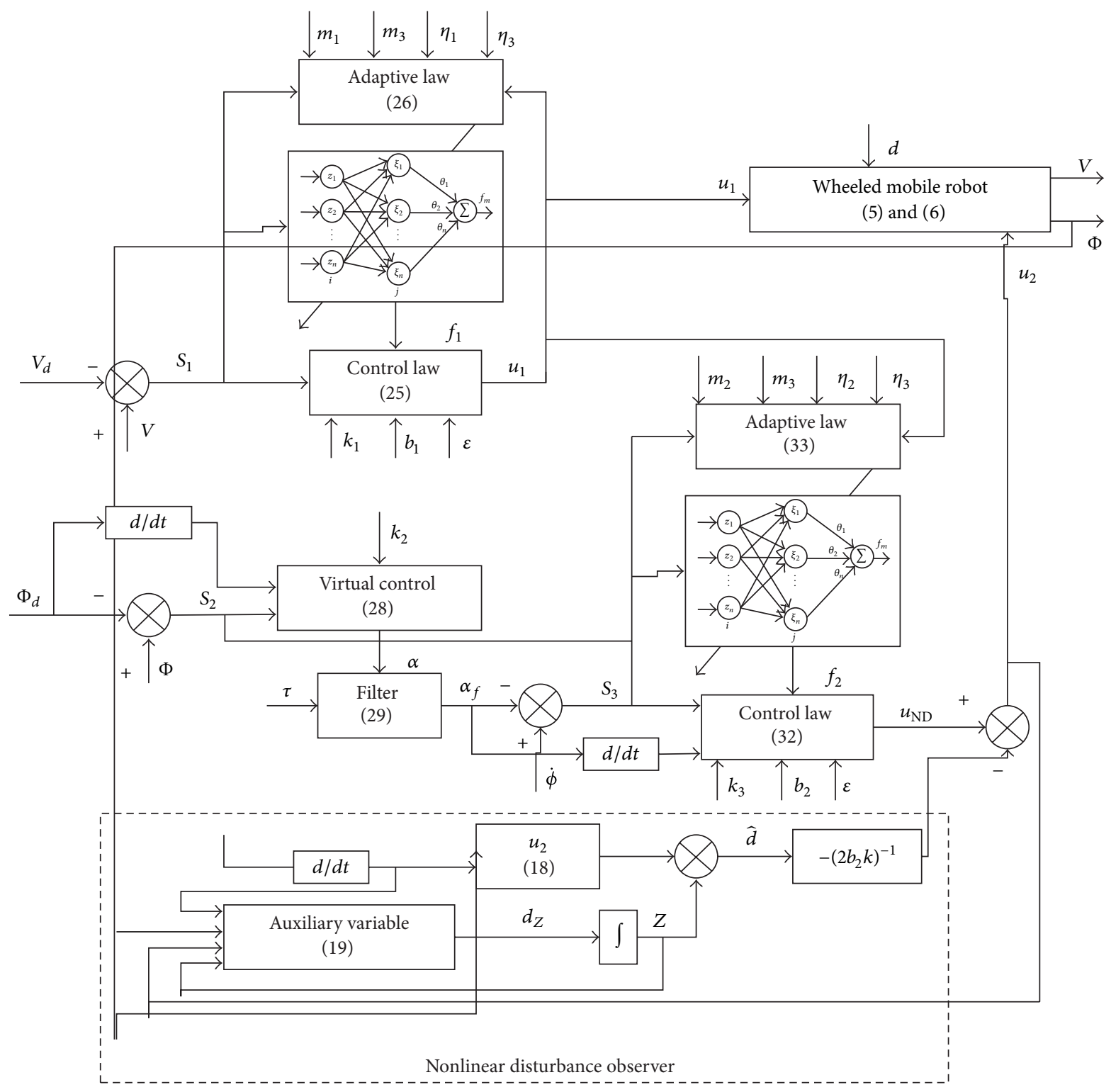

FIGURE 3: Control schematic of wheeled mobile robot.

Step 3. From (30), the corresponding control law is gotten as

$$
u_{\mathrm{ND}}=\frac{1}{2 b_{2} k}\left(k_{3} S_{3}+a_{2} c x_{3}+b_{2} k u_{1}+\widetilde{d}-\dot{\alpha}_{f}\right) .
$$

So far, in contrast to (25) and (32) which belonged to neural network dynamics surface controller, it is easy to see that (34) and (35) which belonged to traditional dynamics surface controller not only need more precise mathematical model but also require more items of expression and coupling items.

\section{Stability Analysis}

Define the filter error $y=\alpha_{f}-\alpha$. Differentiating it results in the following differential equation:

$$
\dot{\alpha}_{f}=-\frac{y}{\tau} .
$$

Introduce boundary layer differential equation as

$$
\dot{y}=-\frac{y}{\tau}+B\left(S_{2}, S_{3}, y, \widehat{k}, \widehat{\theta}_{2}, y_{r}, \dot{y}_{r}, \ddot{y}_{r}\right)
$$

with $B=k_{2} \dot{S}_{2}-\ddot{y}_{r}$ and

$$
y \dot{y} \leq-\frac{y^{2}}{\tau}+y^{2}+\frac{1}{4} B^{2} .
$$

With (24) and (25), (23) can be expressed as

$$
\begin{aligned}
\dot{S}_{1} & =\theta_{1}^{T} \xi_{1}+\delta_{1}-b_{1} \widetilde{k} u_{1}+b_{1} \widehat{k} u_{1} \\
& =\theta_{1}^{T} \xi_{1}+\delta_{1}-b_{1} \widetilde{k} u_{1}+\left(1-\frac{\varepsilon}{\widehat{k}^{2}+\varepsilon}\right)\left(-\widehat{\theta}_{1}^{T} \xi_{1}-k_{1} S_{1}\right)
\end{aligned}
$$




$$
\begin{aligned}
& =-k_{1} S_{1}-\widetilde{\theta}_{1}^{T} \xi_{1}+\delta_{1}-b_{1} \tilde{k} u_{1}+\frac{\varepsilon}{\widetilde{k}^{2}+\varepsilon}\left(\widehat{\theta}_{1}^{T} \xi_{1}+k_{1} S_{1}\right) \\
& \leq-k_{1} S_{1}-\widetilde{\theta}_{1}^{T} \xi_{1}-b_{1} \widetilde{k} u_{1}+\gamma_{1},
\end{aligned}
$$

where $\gamma_{1}\left(S_{1}, \widehat{k}, \widehat{\theta}_{1}, y_{d}, \dot{y}_{d}\right)$ is continuous function and satisfies $\gamma_{1} \geq\left|\delta_{1}+\left(\varepsilon /\left(\hat{k}^{2}+\varepsilon\right)\right)\left(\hat{\theta}_{1}^{T} \xi_{1}+k_{1} S_{1}\right)\right|$.

In the same way, the following can be deduced:

$$
\begin{gathered}
\dot{S}_{2}=S_{3}+y-k_{2} S_{2}, \\
\dot{S}_{3}=\theta_{2}^{T} \xi_{2}+\delta_{2}+b_{2} k u_{1}+2 b_{2} \tilde{k} u_{\mathrm{ND}}-\dot{\alpha}_{f}-2 b_{2} \widehat{k} u_{\mathrm{ND}} \\
=\theta_{2}^{T} \xi_{2}+\delta_{2}+b_{2} k u_{1}+2 b_{2} \tilde{k} u_{\mathrm{ND}}-\dot{\alpha}_{f} \\
-\left(1-\frac{\varepsilon}{\widehat{k}^{2}+\varepsilon}\right)\left(\widehat{\theta}_{2}^{T} \xi_{2}+k_{3} S_{3}+b_{2} \widehat{k} u_{1}-\dot{\alpha}_{f}\right) \\
\leq-\widetilde{\theta}_{2}^{T} \xi_{2}-b_{2} \tilde{k} u_{1}+2 b_{2} \widetilde{k} u_{\mathrm{ND}}-k_{3} S_{3}+\gamma_{2},
\end{gathered}
$$

where $\gamma_{2}\left(S_{2}, S_{3}, \widehat{k}, \hat{\theta}_{2}, y_{r}, \dot{y}_{r}\right)$ is continuous function and satisfies $\gamma_{2} \geq\left|\delta_{2}+\left(\varepsilon /\left(\widehat{k}^{2}+\varepsilon\right)\right)\left(\widehat{\theta}_{2}^{T} \xi_{2}+k_{3} S_{3}+b_{2} \widehat{k} u_{1}-\dot{\alpha}_{f}\right)\right|$.

According to Young's inequality, the calculation produces the following equality:

$$
S_{1} \dot{S}_{1} \leq\left(-k_{1}+1\right) S_{1}^{2}-\widetilde{\theta}_{1}^{T} \xi_{1} S_{1}-b_{1} \tilde{k} u_{1} S_{1}+\frac{1}{4} \gamma_{1}^{2}
$$

In the same way, it can be deduced that

$$
\begin{aligned}
S_{2} \dot{S}_{2} & =\frac{1}{4} S_{3}^{2}+\frac{1}{4} y^{2}-\left(k_{2}-2\right) S_{2}^{2}, \\
S_{3} \dot{S}_{3} \leq & -\widetilde{\theta}_{2}^{T} \xi_{2} S_{3}-b_{2} \widetilde{k} u_{1} S_{3}+2 b_{2} \tilde{k} u_{\mathrm{ND}} S_{3} \\
& -\left(k_{3}-1\right) S_{3}^{2}+\frac{1}{4} \gamma_{2}^{2} .
\end{aligned}
$$

For any given $p>0$, the sets can be defined:

$$
\begin{gathered}
\Omega_{1}=\left\{\left(S_{1}, \widehat{k}, \widehat{\theta}_{1}\right): S_{1}^{2}+\eta_{3}^{-1} \widetilde{k}_{2}^{2}+\eta_{1}^{-1} \widetilde{\theta}_{1}^{T} \widetilde{\theta}_{1} \leq 2 p\right\}, \\
\Omega_{2}=\left\{\left(S_{1}, S_{2}, \widehat{k}, \widetilde{\theta}_{1}\right): \sum_{i=1}^{2} S_{i}^{2}+\eta_{3}^{-1} \widetilde{k}_{2}^{2}+\eta_{1}^{-1} \widetilde{\theta}_{1}^{T} \widetilde{\theta}_{1} \leq 2 p\right\}, \\
\Omega_{3}=\left\{\left(S_{1}, S_{2}, S_{3}, \widehat{k}, \widehat{\theta}_{1}, \widehat{\theta}_{2}, y\right): \sum_{i=1}^{3} S_{i}^{2}+\eta_{3}^{-1} \widetilde{k}_{2}^{2}\right. \\
\left.+\eta_{1}^{-1} \widetilde{\theta}_{1}^{T} \widetilde{\theta}_{1}+\eta_{2}^{-1} \widetilde{\theta}_{2}^{T} \widetilde{\theta}_{2}+y^{2} \leq 2 p\right\} .
\end{gathered}
$$

Theorem 4. To aim at the wheeled mobile robot expressed in (3), satisfying Assumption 2 and giving a positive number $p$, for all satisfying initial condition $V(0) \leq p$, the controllers
(25) and (32) and the adaptive laws (26) and (33) guarantee semiglobal uniform ultimate boundedness of closed loop system with tracking error converging to zero.

Proof. Choose the Lyapunov function candidate as

$$
V=\frac{1}{2}\left(\sum_{i=1}^{3} S_{i}^{2}+y^{2}+\sum_{i=1}^{2} \eta_{i}^{-1} \widetilde{\theta}_{i}^{T} \widetilde{\theta}_{i}+\eta_{3}^{-1} \widetilde{k}^{2}\right) .
$$

Combining (26) and (33) and differentiating (44) give

$$
\begin{aligned}
\dot{V} \leq & \left(-k_{1}+1\right) S_{1}^{2}-\left(k_{2}-2\right) S_{2}^{2}-\left(k_{3}-\frac{5}{4}\right) S_{3}^{2} \\
& +\sum_{i=1}^{2} \frac{1}{4} \gamma_{i}^{2}+\left(\frac{5}{4}-\frac{1}{\tau}\right) y^{2}+\frac{1}{4} B^{2}-b_{1} \widetilde{k} u_{1} S_{1}-b_{2} \widetilde{k} u_{1} S_{3} \\
& +2 b_{2} \widetilde{k} u_{\mathrm{ND}} S_{3}+\widetilde{k} S_{1} u_{1}-m_{3} \tilde{k} \widehat{k}-\sum_{i=1}^{2} m_{i} \widetilde{\theta}_{i}^{T} \widehat{\theta}_{i} .
\end{aligned}
$$

Remark 5. Consider that $-m_{i} \widetilde{\theta}_{i}^{T} \widehat{\theta}_{i}=-m_{i} \widetilde{\theta}_{i}^{T}\left(\widetilde{\theta}_{i}+\theta_{i}\right) \leq$ $-2^{-1} m_{i}\left\|\widetilde{\theta}_{i}\right\|^{2}+2^{-1} m_{i}\left\|\theta_{i}\right\|^{2}, i=1,2$, and $-m_{3} \widetilde{k} \widehat{k} \leq-2^{-1} m_{3} \widetilde{k}^{2}+$ $2^{-1} m_{3} k^{2}$.

Then it can be verified easily that

$$
\begin{aligned}
\dot{V} & \leq\left(-k_{1}+1\right) S_{1}^{2}-\widetilde{k} u_{1}\left(b_{2} S_{3}+b_{1} S_{1}\right)+\sum_{i=1}^{2} \frac{1}{4} \gamma_{i}^{2}-\left(k_{2}-2\right) S_{2}^{2} \\
& +2 b_{2} \widetilde{k} u_{\mathrm{ND}} S_{3}-\left(k_{3}-\frac{5}{4}\right) S_{3}^{2}+\left(\frac{5}{4}-\frac{1}{\tau}\right) y^{2}+\frac{1}{4} B^{2} \\
& +\widetilde{k} S_{1} u_{1}-\frac{1}{2}\left(m_{1}\left\|\widetilde{\theta}_{1}\right\|^{2}+m_{2}\left\|\widetilde{\theta}_{2}\right\|^{2}+m_{3} \widetilde{k}^{2}\right) \\
& +\frac{1}{2}\left(m_{1}\left\|\theta_{1}\right\|^{2}+m_{2}\left\|\theta_{2}\right\|^{2}+m_{3} k^{2}\right) .
\end{aligned}
$$

If $V(t)=(1 / 2)\left(\sum_{i=1}^{3} S_{i}^{2}+y^{2}+\sum_{i=1}^{2} \eta_{i}^{-1} \widetilde{\theta}_{i}^{T} \widetilde{\theta}_{i}+\eta_{3}^{-1} \widetilde{k}^{2}\right)=p$, then $\gamma_{i}^{2} \leq \Delta_{i}^{2}$ and $B^{2} \leq \Gamma^{2}$. Define $\mu=2^{-1}\left(m_{1}\left\|\theta_{1}\right\|^{2}+m_{2}\left\|\theta_{2}\right\|^{2}+\right.$ $\left.m_{3} k^{2}\right)+\sum_{i=1}^{2}(1 / 4) \Delta_{i}^{2}+(1 / 4) \Gamma^{2}$.

Taking those into account, there exists

$$
\dot{V}(t) \leq-2 a_{0} V(t)+\mu .
$$

In the condition of $V(t)=p$, just choosing the appropriate design constant can guarantee $a_{0} \geq \mu / 2 p$, and then $\dot{V}(t) \leq 0 . V(t) \leq p$ belongs to invariant set. From (47), we have

$$
0 \leq V(t) \leq \frac{\mu}{2 a_{0}}+\left(V(0)-\frac{\mu}{2 a_{0}}\right) e^{-2 a_{0} t} \text {. }
$$

\section{Simulation Analysis}

In this section, in order to illustrate the effectiveness of wheeled mobile robot neural network dynamic surface control method which was based on nonlinear disturbance observer, the simulation will be conducted under the initial condition of $x_{1}=x_{2}=x_{3}=0.2$. At the same time, in order to 


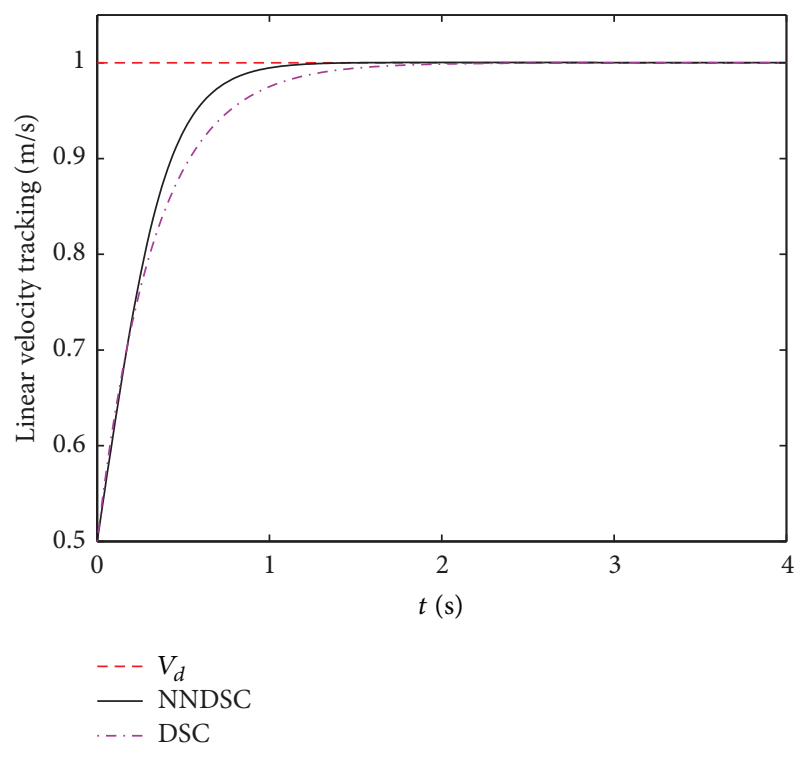

FIGURE 4: Linear velocity trajectory tracking.

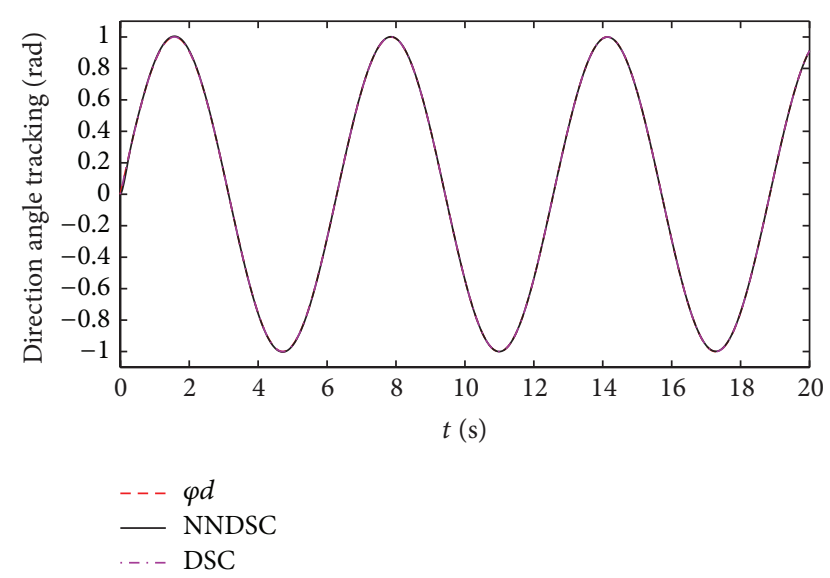

FIGURE 5: Orientation angle trajectory tracking.

give the further comparison, the traditional dynamic surface control will be also used to control the real robot system.

The reference signals are taken as $\varphi(t)=\sin t$ and $v_{d}(t)=$ $1 \mathrm{~m} / \mathrm{s}$ with initial condition $\varphi(0)=0 \mathrm{rad}$ and $v_{d}(0)=0.5 \mathrm{~m} / \mathrm{s}$.

The proposed neural network dynamic surface controllers are used to control the wheeled mobile robot. The center of neural network $S_{i}(z)$ is uniformly distributed in the field of $[-5,5]$, and its width $\sigma_{i}$ is 1.5 . The control parameters are chosen as follows:

$$
\begin{array}{r}
k_{1}=20, \quad k_{2}=20, \quad k_{3}=20, \quad m_{1}=0.06, \\
m_{2}=0.06, \quad m_{3}=0.05, \quad \eta_{1}=1.8, \\
\eta_{2}=1.8, \quad \eta_{3}=1.2, \quad \varepsilon=0.01, \quad \tau=0.01 .
\end{array}
$$

The control parameters of traditional dynamic surface controllers are chosen as follows:

$$
k_{1}=4, \quad k_{2}=4, \quad k_{3}=12, \quad \tau=0.01 .
$$

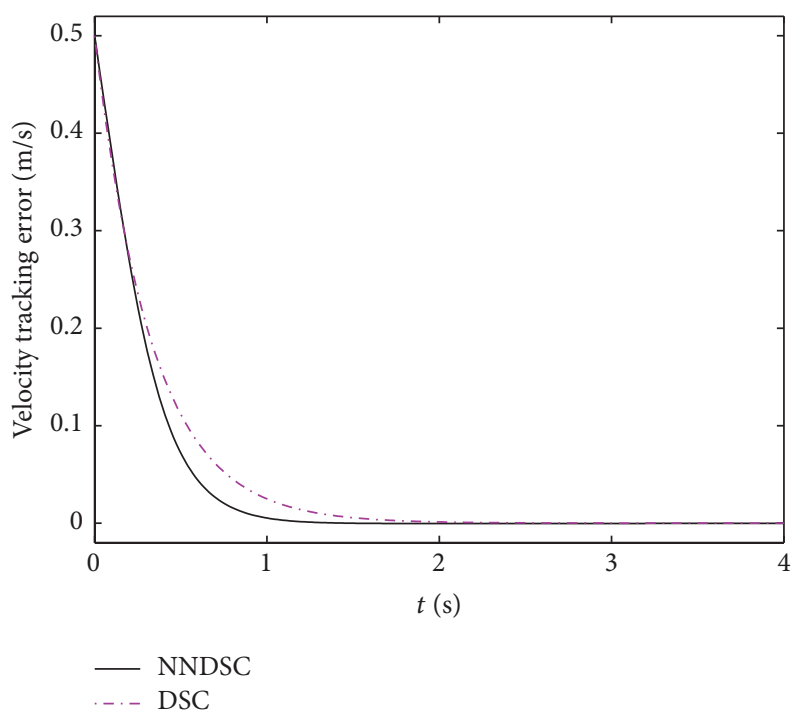

FIGURE 6: Linear velocity tracking error.

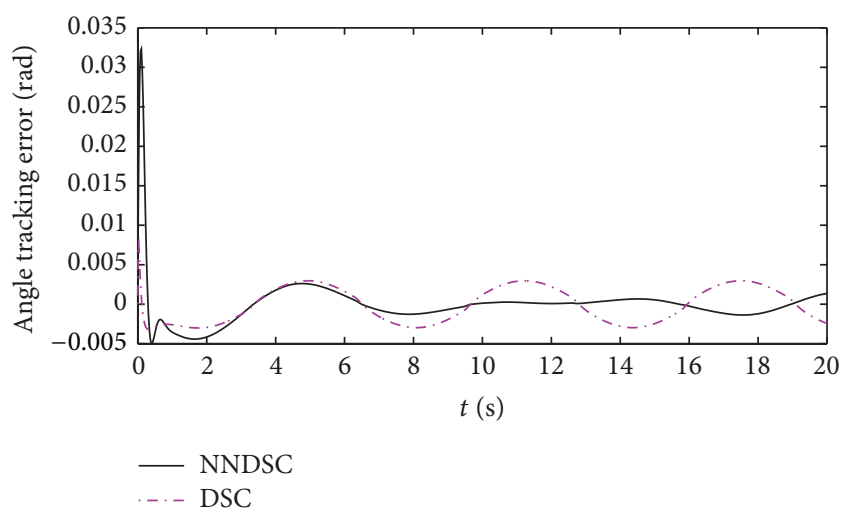

FIGURE 7: Orientation angle tracking error.

In comparison with traditional dynamic surface control, the neural network dynamic surface control is run under the assumption that the system parameters and the nonlinear functions are unknown.

5.1. Trajectory Tracking Analysis. Figures 4-7 display the result of trajectory tracking and tracking error of traditional dynamic surface controller and neural network dynamic surface controller. Figure 4 and Figure 6 show that the response time of NNDSC and DSC are $1.2 \mathrm{~s}$ and $2.2 \mathrm{~s}$, respectively; it is obvious that NNDSC is superior to DSC in the way of convergence velocity. Figures 5 and 7 display that the steady state error of NNDSC and DSC are \pm 0.0003 and \pm 0.003 , respectively; it is clear to see that NNDSC is better than DSC in terms of orientation angle sine tracking.

5.2. Robustness Analysis. As explained in the previous sections, to obtain a precise mathematical model of robot is very difficult and sometimes impossible because of the existence of gear clearance, friction coefficient variation of roadbed, 


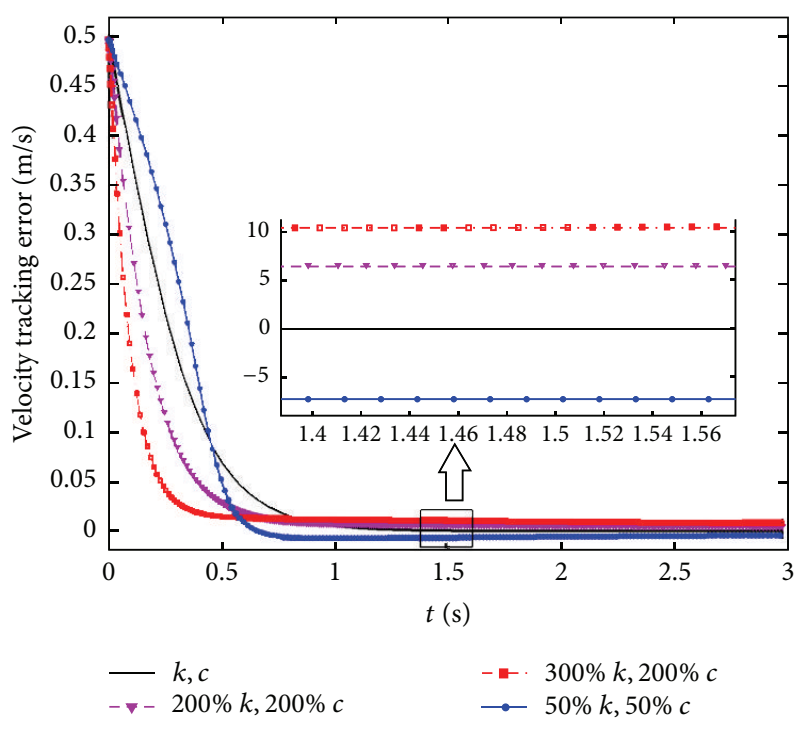

Figure 8: Robustness analysis.

and motor parameter change coming from outside. In such cases, to verify the robustness of system, the drive gain $k$ and friction coefficient $c$ increase by 2 and 3 times, and reduce by half, respectively. Figure 8 presents the tracking error that it is $0.007 \mathrm{~m} / \mathrm{s}$ as $200 \%, 0.011 \mathrm{~m} / \mathrm{s}$ as $300 \%$, and $0.008 \mathrm{~m} / \mathrm{s}$ as $50 \%$. In conclusion, NNDSC can remain always well tuned online and maintain the desired performance according to the variation of environment and still exhibits better robustness and adaptive ability.

5.3. External Friction Disturbance. To take into account Coulomb friction and viscous friction, the external friction of system is given below:

$$
F(v)=F_{c} \operatorname{sgn}(v)+B v
$$

where $F_{c}$ stands for the coulomb friction, $B$ denotes the viscous friction coefficient, and $V$ is the velocity. The corresponding parameters are chosen as $F_{c}=1.5 \mathrm{~N}$ and $B=0.8$.

The nonlinear disturbance observer parameters are taken as $c_{1}=20$ and $c_{2}=20$.

External friction is applied to determine the antidisturbance performance of robot. Figure 9 shows the comparison between real friction and observed friction with nonlinear disturbance observer; it is clearly known that nonlinear disturbance observer observes the change of real friction as well. Figure 10 depicts tracking error of three control algorithms above. The errors of DSC, NNDSC, and NNDSC with nonlinear observer are mean $\pm 0.0695 \mathrm{rad}, \pm 0.0175 \mathrm{rad}$ and $\pm 0.0023 \mathrm{rad}$, respectively. It can be seen that NNDSC with nonlinear observer compensates for the external friction disturbance in finite time duration and makes the system performance better compared to the conventional DSC and NNDSC.

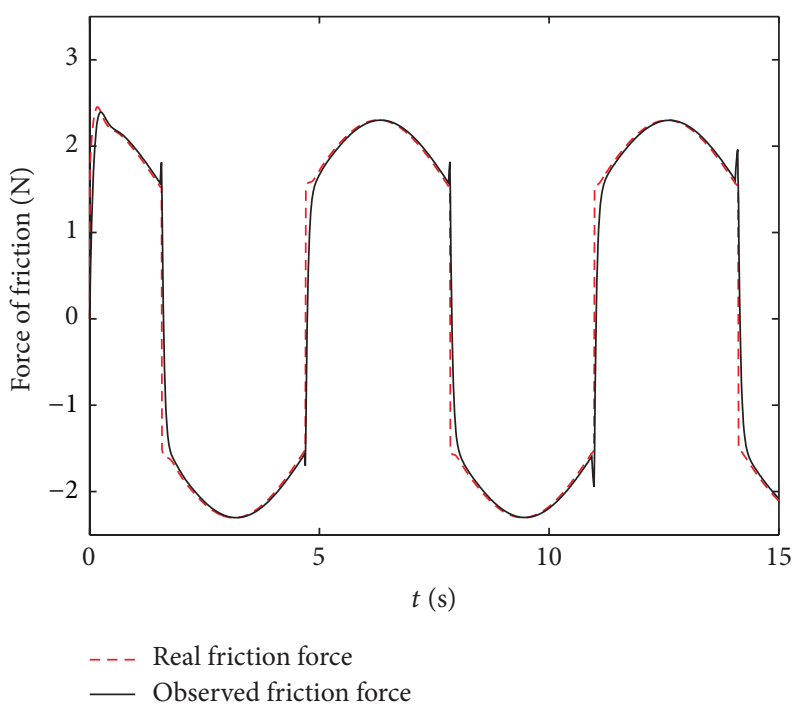

FIgURE 9: Comparison between real friction and observed friction with nonlinear observer.

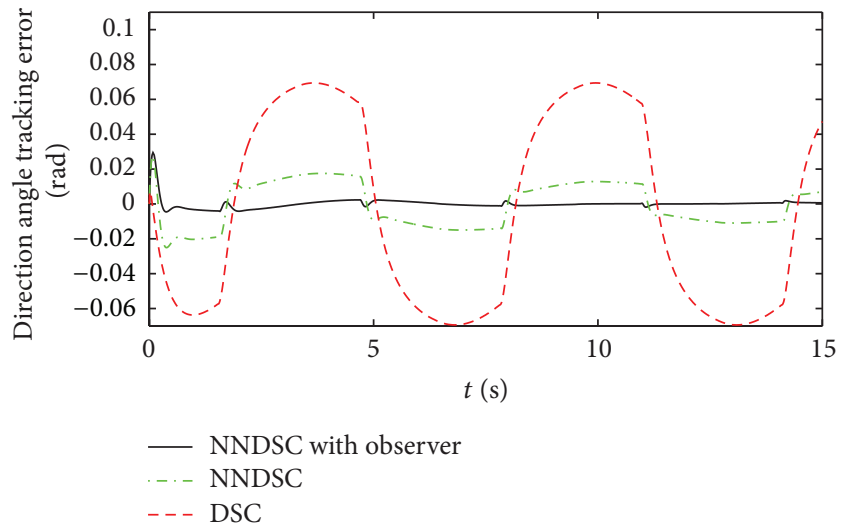

FIGURE 10: Orientation angle tracking error comparison.

\section{Conclusion}

This paper presents an adaptive neural network DSC algorithm based on disturbance observer for uncertain nonlinear wheeled mobile robot system. The presented controller which surmounts the shortages of the conventional DSC guarantees the convergence of tracking error and the boundedness of all the closed-loop signals. In addition, the simulation results are obtained to prove the effectiveness and robustness against the parameter uncertainties and external friction disturbances. Therefore, these characteristics of NNDSC with nonlinear observer show great advantages over the other two methods and make it a competitive control choice for the wheeled mobile robot application.

\section{Conflict of Interests}

The authors declare that there is no conflict of interests regarding the publication of this paper. 


\section{References}

[1] C.-Y. Chen, T.-H. S. Li, Y.-C. Yeh, and C.-C. Chang, "Design and implementation of an adaptive sliding-mode dynamic controller for wheeled mobile robots," Mechatronics, vol. 19, no. 2, pp. 156-166, 2009.

[2] M. Deng, A. Inoue, K. Sekiguchi, and L. Jiang, "Two-wheeled mobile robot motion control in dynamic environments," Robotics and Computer-Integrated Manufacturing, vol. 26, no. 3, pp. 268-272, 2010.

[3] W. Dong and K.-D. Kuhnert, "Robust adaptive control of nonholonomic mobile robot with parameter and nonparameter uncertainties," IEEE Transactions on Robotics, vol. 21, no. 2, pp. 261-266, 2005.

[4] C. De Sousa Jr., E. M. Hemerly, and R. K. Harrop Galvão, "Adaptive control for mobile robot using wavelet networks," IEEE Transactions on Systems, Man, and Cybernetics B, vol. 32, no. 4, pp. 493-504, 2002.

[5] D. K. Chwa, "Sliding-mode tracking control of nonholonomic wheeled mobile robots in polar coordinates," IEEE Transactions on Control Systems Technology, vol. 12, no. 4, pp. 637-644, 2004.

[6] D. Gu and H. Hu, "Receding horizon tracking control of wheeled mobile robots," IEEE Transactions on Control Systems Technology, vol. 14, no. 4, pp. 743-749, 2006.

[7] R. Martínez, O. Castillo, and L. T. Aguilar, "Optimization of interval type-2 fuzzy logic controllers for a perturbed autonomous wheeled mobile robot using genetic algorithms," Information Sciences, vol. 179, no. 13, pp. 2158-2174, 2009.

[8] W. B. J. Hakvoort, R. G. K. M. Aarts, J. van Dijk, and J. B. Jonker, "Lifted system iterative learning control applied to an industrial robot," Control Engineering Practice, vol. 16, no. 4, pp. 377-391, 2008.

[9] J. Ye, "Adaptive control of nonlinear PID-based analog neural networks for a nonholonomic mobile robot," Neurocomputing, vol. 71, no. 7-9, pp. 1561-1565, 2008.

[10] K. Samsudin, F. A. Ahmad, and S. Mashohor, "A highly interpretable fuzzy rule base using ordinal structure for obstacle avoidance of mobile robot," Applied Soft Computing Journal, vol. 11, no. 2, pp. 1631-1637, 2011.

[11] T. Fukao, H. Nakagawa, and N. Adachi, "Adaptive tracking control of a nonholonomic mobile robot," IEEE Transactions on Robotics and Automation, vol. 16, no. 5, pp. 609-615, 2000.

[12] P. Patrick Yip and J. Karl Hedrick, "Adaptive dynamic surface control: a simplified algorithm for adaptive backstepping control of nonlinear systems," International Journal of Control, vol. 71, no. 5, pp. 959-979, 1998.

[13] J. Na, X. Ren, G. Herrmann, and Z. Qiao, "Adaptive neural dynamic surface control for servo systems with unknown deadzone," Control Engineering Practice, vol. 19, no. 11, pp. 1328-1343, 2011.

[14] Q. Chen, X. Ren, and J. A. Oliver, "Identifier-based adaptive neural dynamic surface control for uncertain DC-DC buck converter system with input constraint," Communications in Nonlinear Science and Numerical Simulation, vol. 17, no. 4, pp. 1871-1883, 2012.

[15] T. P. Zhang and S. S. Ge, "Adaptive dynamic surface control of nonlinear systems with unknown dead zone in pure feedback form," Automatica, vol. 44, no. 7, pp. 1895-1903, 2008.

[16] X.-Y. Zhang and Y. Lin, "A robust adaptive dynamic surface control for nonlinear systems with hysteresis input," Acta Automatica Sinica, vol. 36, no. 9, pp. 1264-1271, 2010.
[17] C.-S. Liu and H. Peng, "Disturbance estimation based tracking control for a robotic manipulator," in Proceedings of the American Control Conference, vol. 1, pp. 92-96, June 1997.

[18] C. Zhongyi, S. Fuchun, and C. Jing, "Disturbance observerbased robust control of free-floating space manipulators," IEEE Systems Journal, vol. 2, no. 1, pp. 114-119, 2008.

[19] X. G. Yan, S. Spurgeon, and C. Edwards, "State and parameter estimation for nonlinear delay systems using sliding mode techniques," IEEE Transactions on Automatic Control, vol. 58, no. 4, pp. 1023-1029, 2013.

[20] X. G. Yan, J. Lam, H. S. Li, and I. M. Chen, "Decentralized control of nonlinear large-scale systems using dynamic output feedback," Journal of Optimization Theory and Applications, vol. 104, no. 2, pp. 459-475, 2000.

[21] S. I. Han and K. S. Lee, "Robust friction state observer and recurrent fuzzy neural network design for dynamic friction compensation with backstepping control," Mechatronics, vol. 20, no. 3, pp. 384-401, 2010.

[22] A. Mohammadi, M. Tavakoli, H. J. Marquez, and F. Hashemzadeh, "Nonlinear disturbance observer design for robotic manipulators," Control Engineering Practice, vol. 21, no. 3, pp. 253-267, 2013. 


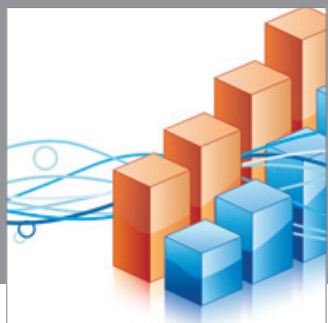

Advances in

Operations Research

mansans

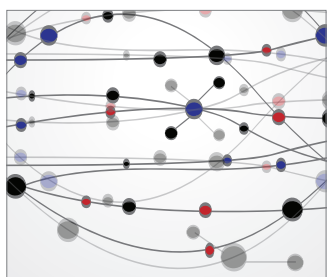

The Scientific World Journal
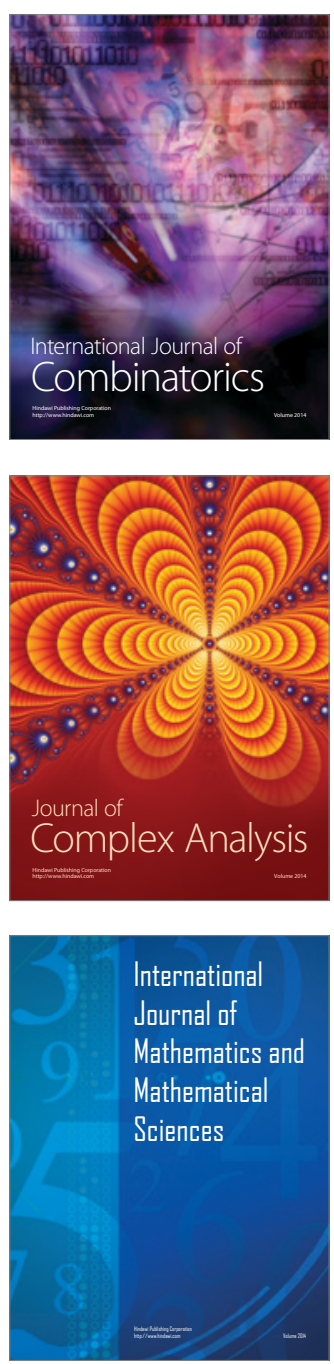
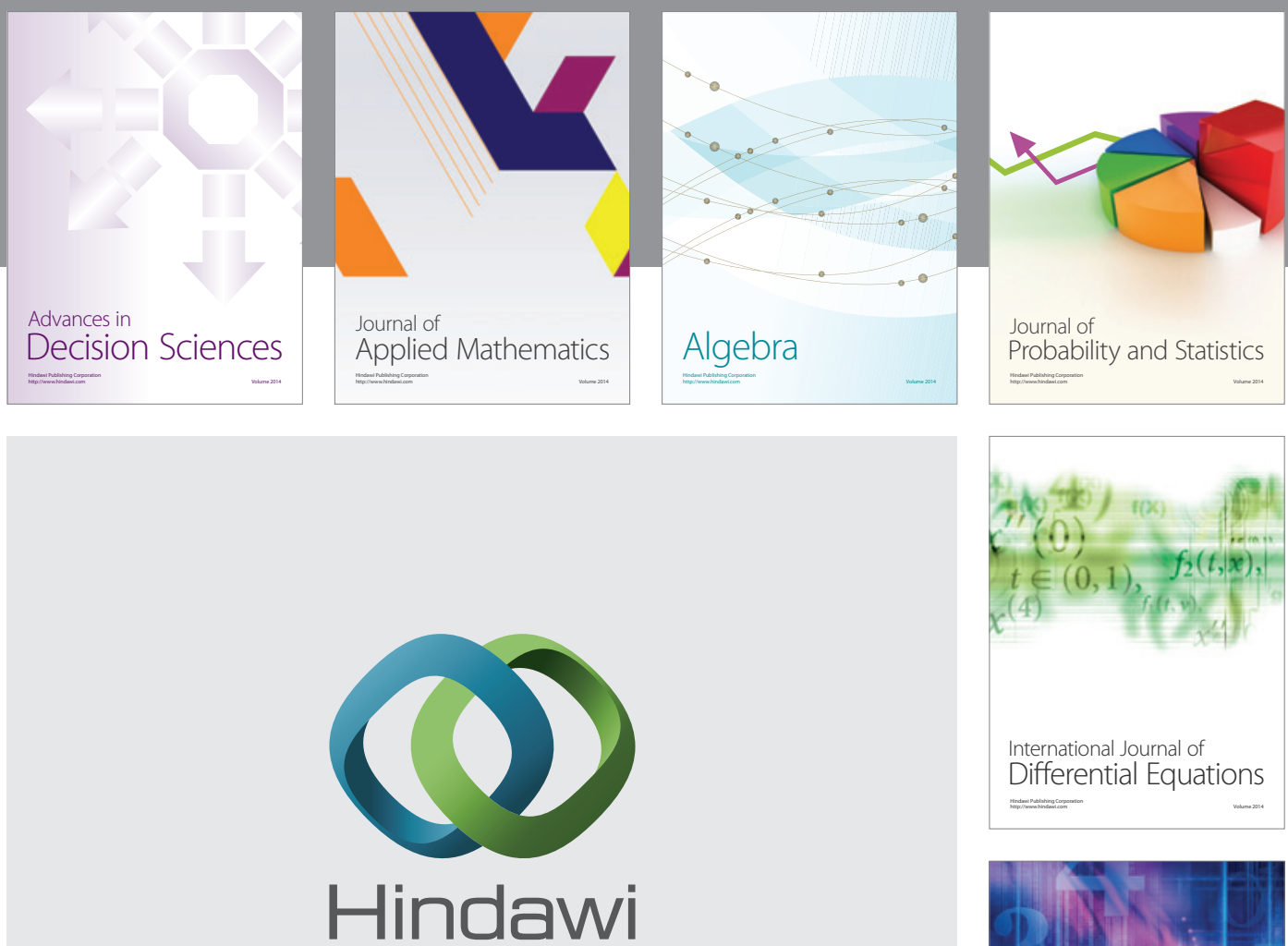

Submit your manuscripts at http://www.hindawi.com
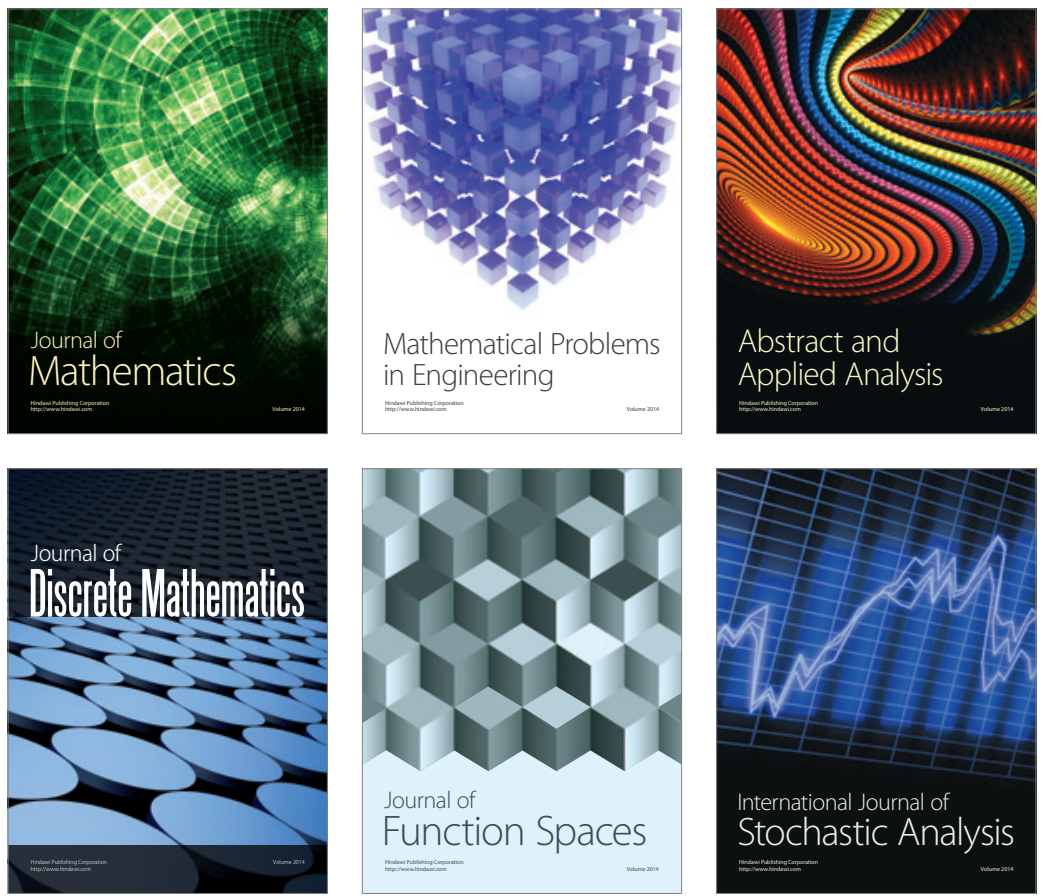

Journal of

Function Spaces

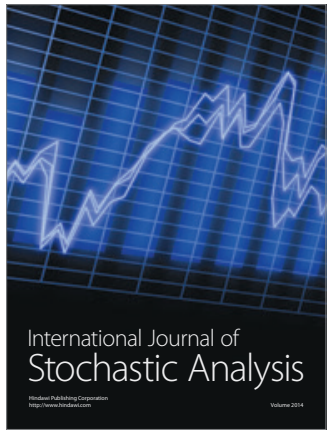

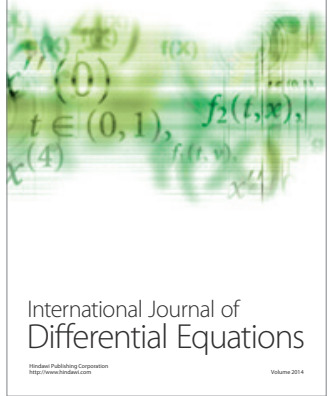
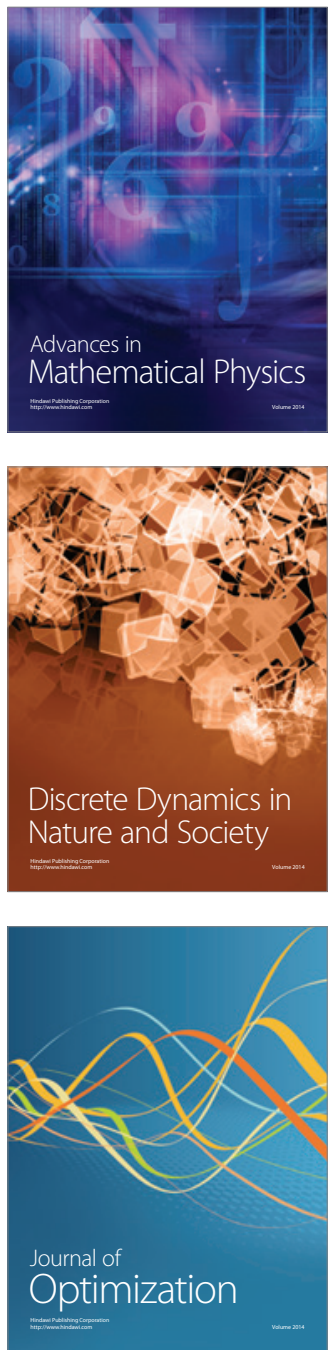